\title{
De la parole à la langue de bois : linguistique théorique et écrits profanes
}

Original Study

Isabelle Morillon

Université de Bourgogne Franche-Comté, Laboratoire CPTC, France.

isabelle.morillon@u-bourgogne.fr

Received: July 2021; Accepted: November 2021

Résumé : Dans cet article, nous proposons d'explorer la notion de parole dans une double perspective : celle de la linguistique de Gustave Guillaume et celle des auteurs profanes Jacques Ellul et Armand Robin. Ces trois auteurs conjugués permettent tout d'abord de déplacer la traditionnelle distinction entre langue et discours dans un cadre englobant linguistique théorique et vision de la langue chez des auteurs non-linguistes. Ensuite, la relation subtile entre parole et société qui se dessine sous la plume critique d'écrivains francophones permet d'envisager la notion de langue de bois par-delà les oppositions habituelles. Dans un premier temps, retracer les trajectoires conceptuelles des notions de langue et de discours nous conduit à identifier la relation entre sens et sujet parlant comme le point de convergence de ces deux trajectoires. Nous montrons ensuite comment la linguistique guillaumienne s'inscrit au cœur de cette convergence en abordant plus particulièrement la définition guillaumienne de l'acte de langage. Enfin, les commentaires de Robin et Ellul sont analysés afin de montrer la convergence des regards sur la notion de parole entre sciences du langage et critique profane.

Mots-clefs : parole, linguistique guillaumienne, langue de bois, dichotomie langue/discours, acte de langage.

\section{LANGUE ET DISCOURS : \\ TRAJECTOIRES THÉORIQUES}

La nécessité de fonder la linguistique comme science donna lieu à la nécessaire circonscription du champ scientifique de la linguistique et par là, la définition de son objet. En tant que science, fondée sur le modèle des sciences humaines en particulier, la linguistique se devait de décrire la langue et non pas la prescrire. Aussi son objet ne pouvait-il être que phénomène soustrait à l'éphémère et à l'insondable subjectivité. Pour le dire en d'autres mots, la linguistique prenait pour objet les moyens du dire dans la langue plutôt que dans l'esprit du sujet parlant. Ce faisant, la linguistique s'arrêtait à la limite de la science de l'homme, là où la philosophie et la métaphysique investiguaient alors. Avec les apports de la psychologie ${ }^{1}$ et ceux de la philosophie de l'esprit, avec ce que l'on pourrait qualifier à la fois de tendance scientifique (la compréhension des mécanismes de la pensée) et philosophique (l'homme et son temps, la vie de l'homme en tant que sujet mû par sa volonté propre), pour ne pas dire sociologique, la linguistique a connu des transformations dans son champ théorique. La question du sens a considérablement élargi le champ de la linguistique, ouvrant la voie à des zones de partage et parfois de conflit avec la sémantique, la sémiotique, et la sociologie. Ce qui fait sens dans le langage, au-delà de la dicibilité théorique, par-delà la logique formelle d'un fait de langue, ne pouvait être appréhendé autrement qu'en intégrant la subjectivité et le contexte, c'est-à-dire la place du sujet en tant qu'être affectif et pensant dans une société faite d'êtres parlant, ou plutôt, discourant. La linguistique de discours s'inscrivit naturellement dans

1 C'est dans la revue Journal de Psychologie que le célèbre article de Benveniste « De la subjectivité dans le langage » fut publié pour la première fois en 1958. 
cette trajectoire. Toutefois, la linguistique de discours est corrélative de la linguistique de langue. C'est-à-dire qu'elle ne la contredit pas mais la reconnait pour mieux la compléter. Autrement dit, la linguistique de discours, bien qu'ultérieure à la linguistique moderne initiale, confirme la dichotomie langue-discours. Nous pourrions même dire qu'elle en dépend car sans cette dichotomie, la linguistique de discours ne pourrait pas délimiter son champ d'application.

\section{DE LA STYLISTIQUE À LA} LINGUISTIQUE ÉNONCIATIVE

Si la linguistique de l'énonciation est certainement le courant théorique qui marque le plus nettement l'émergence de la linguistique dite de discours, on peut voir les prémisses d'un changement de regard avant même la publication du Cours en 1916. L'ouvrage de Charles Bally (1865-1947), Le Langage et la vie, dès 1913, inaugure ce que l'auteur appellera la stylistique, fondée sur la dimension affective et subjective du langage. Nous retrouvons dans cet ouvrage, dédié à Louis-Ferdinand de Saussure, la dimension sociale du langage évoquée dans le Cours de linguistique générale. ${ }^{2}$ Bally va cependant plus loin en mettant en relief la valeur pragmatique du langage, instrument d'action et surtout en introduisant la valeur affective du langage, manifestée à l'échelle individuelle : « Le langage naturel et spontané, instrument d'expression et d'action dans la vie réelle, mérite d'être étudié dans ce qui fait son essence, c'est-à-dire son contenu subjectif et affectif, » (Bally 1916, 27). La stylistique de Charles Bally, que l'auteur inscrit dans la continuité de l'œuvre de Saussure avec la distinction entre langue et parole, vise à considérer la langue parlée comme une «province » du domaine de la langue (ibid., 159). Bally distingue la valeur intellectuelle de la langue qui suppose objectivité et logique et vise à mettre en ordre des idées, de la valeur affective du langage spontané, nécessairement parlé, lié à la vie réelle que connaît tout sujet parlant. Ainsi, la volonté d'intégrer pleinement la dimension subjective du langage dans l'étude linguistique est probablement le premier pas vers la linguistique de discours dans sa forme aboutie. La subjectivité dans le langage est également au centre de l'œuvre d'Emile Benveniste qui considère comme réducteur, et par là erroné, de voir le langage comme un instrument extérieur à I'homme, et affirme $(1966,266)$ :
«Bien des notions en linguistique, peut-être-même en psychologie, apparaîtront sous un jour différent si on les rétablit dans le cadre du discours, qui est la langue en tant qu'assumée par l'homme qui parle, et dans la condition d'intersubjectivité, qui seule rend possible la communication linguistique. $»^{3}$

À strictement parler maintenant, la première phase de la linguistique dite de langue à la linguistique dite de discours peut être identifiée au moment de l'émergence de la théorie de l'énonciation dont l'un des linguistes fondateurs est Antoine Culioli (1924-2018) dont les articles furent réunis dans un ouvrage qui fait référence aujourd'hui : Pour une linguistique de l'énonciation. Le travail d'Antoine Culioli s'inscrit dans une démarche critique de la linguistique structurale élaborée à la suite du Cours de Ferdinand de Saussure (bien que Saussure lui-même n'emploie pas le terme structure mais celui de système). La linguistique de l'énonciation, en intégrant la subjectivité du sujet parlant, nommé énonciateur, ouvre la voie à de nouvelles perspectives où l'intention du locuteur et la dimension argumentative du langage prennent toute leur place. Oswald Ducrot s'inscrit également dans le cadre de la linguistique de l'énonciation avec deux notions théoriques majeures : la polyphonie, ${ }^{4}$ tout d'abord, que Mikhail Bakhtine (1895-1975) avait introduite dans le champ du genre romanesque, la notion de présupposé, ensuite, et ses corollaires : le posé et le sous-entendu; notions qui admettent la valeur argumentative du langage à l'œuvre dans le discours.

II convient à présent de s'intéresser à la linguistique de discours dans ses prolongements les plus récents, c'est-à-dire à l'analyse du discours. L'analyse du discours en tant que discipline à part entière est corrélée à l'émergence d'outils et de méthodes d'analyse textuelle, parfois conçus par les linguistes eux-mêmes. L'analyse du discours s'appuie alors sur des traitements de corpus afin de réaliser des études logométriques et textométriques. Ainsi, en tentant de retracer le chemin parcouru à partir de la linguistique structurale du début du siècle jusqu'à l'analyse de discours la plus récente, nous voyons un mouvement partant de la subjectivité, notion qui amène naturellement celle d'acte de langage pour aboutir à la nécessaire prise en compte du sens. En quelque sorte, la linguistique de discours est l'aboutissement d'une réflexion de plus en plus intégrante: de la subjectivité individuelle, tout d'abord, puis, nécessairement, des conditions extérieures de cette subjectivité,

2 II a parfois été reproché à Saussure de ne pas accorder l'importance qu'elle mérite à la dimension sociale de la langue, c'est-à-dire de ne pas étudier la langue en tenant compte du rôle du sujet parlant. Cette critique de la linguistique sensible chez des auteurs comme Pierre Bourdieu aura influencé d'autres interprétations du même ordre notamment outre-Atlantique, en analyse du discours, par exemple chez Norman Fairclough. Cette dimension est pourtant bien évoquée dans le Cours publié en 1916 et loin d'être étudiée comme une entité statique, la langue est considérée comme instrument social en perpétuelle évolution.

3 E. Benveniste, Problèmes de linguistique générale vol. 1. Paris, Gallimard. 1966, p. 266.

4 O. Ducrot, Le Dire et le dit, Paris, Les Éditions de Minuit, 1984. 
c'est-à-dire de l'environnement social, voire politique ${ }^{5}$ qui servent de terrain à l'exercice du langage.

\section{DE LA DIMENSION DU SUJET}

\section{À LA DIMENSION SOCIALE}

L'acte de langage est le moment de basculement, en linguistique occidentale tout du moins, d'une linguistique nécessairement restrictive en quête de cadre, vers une linguistique qui cherche à intégrer les éléments extérieurs à la langue elle-même de façon à mieux cerner la question du sens. Le sens en discours, résume Patrick Charaudeau,

«[...] arrivant par les savoirs qui se construisent dans la pratique sociale, il se trouve ensuite inoculé dans le sens de langue qui après quelques hésitations finit par l'accepter, voire l'intégrer au point, peut-être de se l'approprier et de le catégoriser dans une nouvelle dénotation. $»^{6}$

Ainsi, la linguistique de discours ne saurait être regardée comme une discipline exclusive, dissociée du domaine de la langue et l'on peut voir dans la citation qui précède un raisonnement qui n'est pas sans rappeler certains passages du Cours où sont évoquées les conditions du changement linguistique, changement qui pour réussir suppose l'adoption en discours de nouvelles formes par les sujets parlants. Le discours peut ainsi être compris comme une expression sociale, fruit de l'influence du contexte collectif dans lequel évoluent les individus ; contexte aux facettes culturelles, intellectuelles et politiques d'une époque.

Louis-Jean Calvet (2017) rappelle l'origine de la sociolinguistique dans la pensée d'Antoine Meillet (18661936), avant même la parution du Cours de Ferdinand Saussure. On retrouve en effet chez Meillet l'attention portée sur la subjectivité du sujet parlant et en particulier, la dimension affective du langage. Le célèbre linguiste comparatiste inaugure ainsi une rupture avec la vision héritée de Saussure qui consiste à limiter le champ de la linguistique au seul périmètre de la langue en tant que système général. Le développement de la sociolinguistique en tant qu'approche affirmée interviendra nettement entre les années 1960 et 1970 aux ÉtatsUnis. William Labov (1972) affirmera d'ailleurs que la sociolinguistique est la linguistique ; autrement dit, il ne peut y avoir de linguistique sans prise en compte de la dimension sociale des interactions langagières. La sociolinguistique de Labov devrait toutefois être resituée dans son contexte de l'étude de la variation linguistique en fonction des contextes socioculturels. II s'agit alors d'une approche destinée à poser un cadre théorique et méthodologique propice à l'étude des sociolectes et des situations de diglossie. Néanmoins, on peut voir les prolongements multiples d'une telle approche jusqu'à nos jours avec l'émergence d'une sociolinguistique urbaine, par exemple. Louis-Jean Calvet souligne également l'inspiration marxiste des sociolinguistes de l'époque. Finalement, la sociolinguistique est nettement orientée vers une vision sociologique de l'être parlant où la subjectivité individuelle est conçue en fonction de l'appartenance à un groupe plus vaste de nature culturelle ou sociale, voire de classe à strictement parler. On peut voir dans ce glissement théorique un mouvement du général au particulier allant de la langue en tant que système commun institué et partagé vers une vision particularisante qui cherche la clef de son objet dans un grain de plus en plus fin. Néanmoins, on peut se demander, la question est actuelle, si une telle approche n'aurait pas tendance à faire de la sociologie le terrain englobant de la linguistique. En effet, il semble que la prise en compte de la dimension sociale du sujet parlant, à mesure qu'elle se renforce, aboutisse à une vision fragmentée de la langue. Dans le même temps, le caractère contraignant du système de la langue, caractère commun à toute langue, se déplace vers le caractère contraignant du discours où l'actualisation de la langue est subordonnée à l'appartenance à des groupes particuliers. Aussi, linguistique de discours et sociolinguistique partagent certains fondements théoriques et partent d'un élan commun. La méthode textométrique ainsi que certaines thématiques (nous pensons notamment aux idéologies linguistiques) ${ }^{7}$ sont communes aux deux approches. Toutefois, alors que la linguistique de discours se fonde sur la distinction saussurienne langue-parole, la sociolinguistique cherche à s'en écarter. De plus, alors que la sociolinguistique transpose la contrainte systémique de la langue vers le milieu social, la linguistique de discours s'attache à prendre en compte la part individuelle du langage.

Pour terminer sur la relation entre linguistique de discours et sociolinguistique, nous voyons deux points

5 Nous pensons notamment à Maurice Tournier (1933-2013), fondateur de la revue Mots. Les langages du politique et à Pierre Achard, fondateur de la revue Langage et société.

6 P. Charaudeau, [De la linguistique de la langue à la linguistique du discours, et retour.] In : Engwall, Gunnel \& Fant, Lars (eds.) Festival Romanistica. Contribuciones lingüísticas - Contributions linguistiques - Contributi linguistici Contribuições linguísticas. Stockholm Studies in Romance Languages, Stockholm: Stockholm University Press. 2015, p. 3-12.

7 L'écriture inclusive, à ce titre, et sa réception en sociolinguistique, montre qu'il existe une fracture épistémologique entre linguistique d'inspiration saussurienne, voire littéraire et comparatiste, et linguistique d'inspiration sociologique. II est commun de considérer la critique de l'écriture inclusive comme une forme d'idéologie linguistique. Cependant, en termes saussuriens, c'est l'imposition de l'écriture inclusive qui relève de l'idéologie et ce à plusieurs titres : changer les mots comme moyen de changer la référence et invoquer des instances régulatrices au lieu de laisser l'usage décider. 
d'ancrage : un premier correspond à une linguistique qui tend vers la nécessaire prise en compte du sujet parlant. Le deuxième point d'ancrage est celui de la sociologie, où l'environnement socioculturel devient le paramètre prédominant à toute étude du discours. Le premier point part de la langue en tant que matériau commun pour arriver au sujet dans son unicité et sa volonté d'action. ${ }^{8} \mathrm{La}$ ligne ainsi tracée conduit à une linguistique de discours orientée vers le sujet et non sans lien avec la phénoménologie. Le deuxième point part du sujet parlant pour le réinterpréter à l'aune du groupe social dans lequel il évolue. Dans ce cas, la subjectivité de l'être locuteur est volontiers interprétée en tant qu'idéologie linguistique et la textométrie sert de base méthodologique à l'étude des représentations sociales qui se manifestent dans les discours profanes sur la langue.

La linguistique de discours suppose un sujet énonciateur, la sociolinguistique suppose un environnement conditionnant. Aussi, située en linguistique de discours, une étude des liens entre langage et pouvoir aura tendance à focaliser sur la relation entre subjectivité et signification alors qu'une telle étude située en sociolinguistique aura tendance à rechercher les conditions de production des discours dans leur aspect hétérogène et en s'appuyant sur les notions de contexte et de normes.

Les trajectoires croisées de la linguistique de langue et de la linguistique de discours conduisent à mettre en évidence la tendance naturelle de la linguistique après Saussure d'inclure la subjectivité dans le cadre de son objet. La linguistique guillaumienne, dans sa réception contemporaine, entre tendance mentaliste et pragmatique, propose un modèle conciliant linguistique de langue et linguistique de discours au sein duquel la parole procède des deux champs dans un rapport de successivité. La parole, chez Gustave Guillaume procède à la fois de la genèse du discours que de la langue à l'état de puissance. Qu'elle soit silencieuse, présente à l'état virtuel ou effective, versée en discours, elle est chaque fois nettement corrélée à l'existence d'un sujet parlant et pensant réel.

\section{LE LANGAGE EN ACTES : CE QUE PARLER VEUT DIRE EN LINGUISTIQUE GUILLAUMIENNE}

L'œuvre de Gustave Guillaume (1883-1960) a bénéficié d'un tel regain d'intérêt ces vingt dernières années que les développements théoriques de l'élève de Meillet ont fait l'objet d'une vaste littérature. Gustave Guillaume conçoit l'acte de langage comme un entier au sein duquel faits de langue et faits de discours entretiennent un rapport de successivité. Les actes de langue sont des actes de représentation auxquels succèdent des actes de discours, actes d'expression. Par actes de représentation, il faut entendre les moyens dont dispose la pensée pour se saisir elle-même. La langue est donc le lieu et la condition du pensable alors que le discours est une phase de réalisation individuelle, d'actualisation en contexte. La notion de successivité implique la nécessaire dimension temporelle du passage entre langue et discours. Ce facteur temporel est nommé temps opératif par Gustave Guillaume. Cependant, la question de la réalité de ce temps opératif est primordiale car sur cette base repose la dimension énonciative de la linguistique guillaumienne. Si le discours actualisé est bien le résultat de saisies successives de la pensée, saisies effectuées dans un temps infime et cependant réel, alors la linguistique guillaumienne s'inscrit dans une linguistique énonciative, c'est-à-dire dans une linguistique qui fait le pont entre langue et discours, entre système institué et acte de langage à caractère subjectif. D'autre part, dans la théorie de Gustave Guillaume, les signes ne sont pas considérés comme des contenus toujours disponibles que le locuteur utiliserait en fonction des énoncés qu'il cherche à produire. Les opérations de pensée vers la langue puis vers la réalisation en discours doivent être réitérées pour chaque acte allocutoire. II existe donc deux dimensions dans la linguistique guillaumienne : une dimension cognitive qui s'inscrit dans une linguistique mentaliste, et une dimension énonciative. ${ }^{9}$ La dimension énonciative a été développée par André Joly à la fin des années 1980 et plus récemment par Mathieu Valette et Francis Tollis. Selon ce dernier, la psychomécanique de Gustave Guillaume se prête à une lecture socio-opérative ce qui la rapproche de toutes les linguistiques de discours. La théorie guillaumienne permet en effet d'unifier linguistique de langue et linguistique de discours (que Guillaume qualifiait de linguistique d'aval) autour d'un seul noyau : celui de la subjectivité, c'est-à-dire de la signification.

\section{ACTE DE LANGAGE \\ Le terme acte de langage prend un sens plus ou moins englobant dans la théorie Guillaumienne, en fonction de l'avancée des travaux de l'auteur. Le premier sens est le plus restrictif et fait référence à l'actualisation du système de la langue dans le domaine effectif de la chaîne parlée $(1929,42)$. Autrement dit, l'acte de langage est équivalent à l'acte d'expression (ou acte de discours). La deuxième acception est bien moins restrictive. L'acte de langage correspond alors au contact entre pensée et langue, bien en amont du domaine du discours. Guillaume $(1971,10)$ écrira que l'acte de langage est « l'acte par lequel nous passons de la pensée à son expression au moyen de la}

8 La praxématique peut être située dans cette catégorie dans la mesure où le sens est situé au niveau du sujet individuel mais interprété de façon pragmatique. Le praxème en est l'un des outils conceptuels.

9 Ce sont là deux aspects dont Mathieu Valette explique la présence dans la théorie de Gustave Guillaume. M.Valette, «Énonciation et cognition : deux termes in absentia pour des notions omniprésentes dans l'œuvre de Guillaume, » Le Français moderne, 2003, p. 6-25. 
parole. » II s'agit d'un acte mental, qui ne peut être élucidé par le linguiste. Le terme acte de langage prend ici un sens plus intégral : «Accomplir un acte de langage, c'est [...] transiter du plan de puissance où siège la langue, au plan d'effet où siègera et se formera le discours. » L'acte de langage correspond alors, non plus seulement au résultat des opérations situées en pensée et en langue puis en discours, mais au processus de passage d'un plan à l'autre qui mène à ce résultat. Plus tardivement, à partir des années 1950, Guillaume aura tendance à revoir sa théorie selon une vision plus déterministe de la langue. Il distinguera alors deux phases successives dans l'acte de langage : une phase précoce, l'acte de représentation qui correspond à l'opération constructrice du mot, située en langue, et une phase tardive qui correspond à l'opération constructrice de la phrase, située en discours. Guillaume avancera alors la thèse selon laquelle la langue est naturellement destinée à supporter les opérations de construction du mot de façon à alléger la phase de construction de la phrase. Selon une loi d'économie du langage, il semble que les langues évoluent vers une tendance croissante à porter en système les matériaux constructifs de l'énoncé de façon à alléger la phase de construction du discours, inhérente à l'intention du locuteur.

Nous venons de présenter les trois orientations définitoires de l'acte de langage dans la théorie de Guillaume et nous avons adopté l'ordre chronologique pour souligner l'évolution théorique. Au début de son cheminement, Guillaume donnait une définition restrictive de l'acte de langage, entendu comme acte de discours, et privilégiait alors la dimension pragmatique de l'expression du sujet parlant. En dernier lieu, vers la fin de son œuvre, il semble qu'un changement de paradigme ait eu lieu. Mathieu Valette explique en partie ce changement par l'influence des idées de l'époque, notamment par l'émergence de la cybernétique. II est cependant possible de présenter les différents sens que prend le terme acte de langage de façon différente, en partant de la définition la plus restrictive vers la moins restrictive. Dans un premier temps, l'acte de langage équivaut à l'acte d'expression et est situé strictement en discours. Selon une vision moins restrictive, l'acte de langage est subdivisé en deux phases successives dont l'une des deux seulement concerne le discours. Enfin, l'acte de langage est regardé comme le point charnière entre acte de pensée et acte de discours. Dans tous les cas, qu'il s'agisse de l'acte de langage et ses variantes définitoires, de l'acte de représentation situé en langue ou de l'acte d'expression situé strictement en discours, chaque fois Guillaume emploiera le mot acte, indiquant par-là que le sujet parlant agit à chaque étape du langage.

\section{ACTE DE REPRÉSENTATION ET ACTE D'EXPRESSION}

Selon le modèle guillaumien, l'homme parlant est indissociable de l'homme pensant. Si le langage est la nécessaire condition à la mise en forme de la pensée, réciproquement, la parole suppose l'existence préalable d'une pensée pour lui donner matière. Cependant, la linguistique de Gustave Guillaume est une systématique visant à déterminer les conditions d'expression situées dans le système de la langue et non dans la volonté du sujet. Aussi, on peut obtenir des interprétations différentes de la théorie guillaumienne selon la part que l'on accorde à la notion de choix dans le passage de la pensée à la langue puis au discours. Selon une première lecture, on peut voir dans l'affirmation guillaumienne du lien entre pensée, langue, et discours l'affirmation de la dimension subjective du langage prenant pour centre le locuteur pensant. Cela peut mener à une lecture phénoménologique de la théorie guillaumienne. Toutefois, la systématique du langage comporte un aspect à prendre en compte : celui du système de la langue en tant que cadre de la pensée. Or, au fur à mesure de l'élaboration de sa théorie, Gustave Guillaume aura tendance à attribuer au système de la langue un caractère plus autonome et contraignant. Selon cette tendance tardive, la place de la pensée subjective, nécessairement singulière, sera revue au profit du caractère contraignant du système de la langue. Si la dimension subjective, voire phénoménologique, subsiste dans la théorie guillaumienne, cela tient en grande partie à l'affirmation des opérations d'aperception et de pensée individuelles qui prévalent à l'acte d'expression. En effet, la linguistique guillaumienne ne considère pas les signifiés et les signes (ou signifiants selon la terminologie saussurienne) comme disponibles dans la mémoire du sujet parlant. En d'autres termes, le sujet parlant ne prélève pas les signes dans un vaste répertoire symbolique en vue de faire passer un message à un destinataire. Chaque fois réitéré, l'acte de langage du sujet parlant s'inscrit dans un temps unique où l'acte d'expression se renouvelle selon un processus actualisé par le sujet.

\section{ACTUALISATION : VERS UNE INTERPRÉTATION PRAGMATIQUE DE LA LINGUISTIQUE GUILLAUMIENNE}

Les soubassements théoriques de la linguistique guillaumienne ont été mis à profit par les linguistes qui appelaient de leurs vœux une linguistique de la parole, appelée praxématique, fondée sur le langage en actes plutôt que sur la langue en tant que système.

Gustave Guillaume déclarait lui-même en 1947 (1970, 157) : «Le discours répond à une visée d'effet : engager une opération de discours c'est de toute évidence vouloir agir, produire un effet sur quelqu'un. » La praxématique s'affirme en tant que linguistique de la parole dans un article de Robert Lafont daté de 1983. La notion d'actualisation, attribuée à Bally (1922) et reconnue chez Gustave Guillaume, sert de référence pour montrer que l'étude de la langue ne saurait consister en la description d'un système clos. Lafont confronte une vue idéaliste de la langue fondée sur des modèles abstraits à une vue empiriste fondée sur les réalisations concrètes du langage. La théorie Guillaumienne peut se prêter à des interprétations de types 
pragmatiques et phénoménologiques. Lafont s'appuie sur les silences et les hésitations observés lors du discours pour montrer la réalité du temps opératif, appelé temps de l'« à-dire » (art. cité, 38) : « Nous avons été amenés à placer là la preuve matérielle du temps opératif, joignant ainsi l'expérience de laboratoire à la nécessité théorique apparue à l'intérieur du système explicatif. » Gustave Guillaume disait lui-même (ibid., 157) : « La langue existe en nous alors même que nous ne parlons pas. »Cependant, si la praxématique a le mérite de poser les bases d'une linguistique émancipée du paradigme structuraliste et de la dichotomie langue-parole, il semble nécessaire de modérer sa réception du temps opératif. Ce temps opératif est, chez Gustave Guillaume, un temps nécessaire pour penser avant d'être nécessaire pour parler. La notion d'actualisation, que Guillaume emploie en 1929, est également assez différente en praxématique. Lafont entend par actualisation, tout en faisant référence à la théorie guillaumienne, l'actualisation du sens en discours. II s'agit là de montrer que le sujet parlant actualise la langue dans la parole et que l'étude de la langue dans son usage doit être une étude de la langue actualisée par le sujet parlant.

Cette interprétation est plus proche de ce que développe Bally au sujet de l'ouvrage de Ferdinand Brunot intitulé Langue et pensée dans un article éponyme paru en 1922. Gustave Guillaume $(1929,134)$ écrit : « La langue, en soi, n'exprime rien : elle représente, elle est représentation. L'expression appartient au seul discours qui exprime à partir du représenté et avec les moyens que le représenté offre. » On retrouve ici l'idée de Bally développée dans une longue note au début de son article publié en 1922. La linguistique guillaumienne se veut génétique, c'est-à-dire partant des faits de langue pour remonter aux représentations préalables au système de la langue: «on a pu ainsi étudier les formes dans leur phase génétique, antérieure à leur actualisation dans la parole alors qu'il est de tradition de ne les considérer que dans leur phase de réalité, postérieure à cette actualisation. » Autrement dit, Guillaume visait l'élucidation des opérations qui précèdent l'actualisation. Dans cette mesure, la notion d'actualisation semble peu propice à faire de la linguistique guillaumienne une linguistique de la parole au sens de la praxématique. Cela dit, il est incontestable que la linguistique guillaumienne a obtenu la reconnaissance d'un certain nombre de linguistes soucieux d'utiliser un modèle intégral prenant en compte la dimension du sujet-locuteur et la subjectivité dans le processus de la pensée au discours.

La praxématique offre un exemple incontournable de la réception tardive de la théorie guillaumienne. Cependant, les liens entre théorie de la communication et praxématique semblent encore éloigner cette dernière des fondements guillaumiens. Les codes de la théorie de la communication et de l'approche fonctionnelle du langage se retrouvent dans le texte de Lafont à plusieurs reprises à travers l'emploi des termes « programme, » « code, » «message. » Lafont évoque ainsi (ibid., 38) le « programme de l'à-dire. » Le reproche a souvent été fait à la linguistique de l'École de Genève de se fonder sur un modèle, excluant alors la parole et l'usage réel de la langue par les locuteurs. Ce reproche s'inscrit dans une réaction au positivisme ${ }^{10}$ et à la tendance à partir d'un modèle théorique plutôt que des conditions réelles de manifestation du langage. Cependant, la linguistique saussurienne a au moins le mérite de reconnaître ce qui est insaisissable par la voie scientifique et on peut se demander si le scientisme ne serait pas finalement plus présent chez ceux qui tentent de modéliser le discours lui-même dans sa dimension subjective. En somme, plus la linguistique se veut englobante, plus le modèle doit l'emporter.

Le versant phénoménologique et pragmatique de la linguistique guillaumienne est nuancé par le caractère indépendant de la langue. La distinction entre représentation et expression est fondamentale dans la théorie guillaumienne et il convient d'examiner soigneusement ce qui, de la pensée, de la langue et du discours ressortit à chacun de ces domaines. "Le discours a toujours une visée et c'est celle du sujet parlant. »

Cette phrase capitale que l'auteur de la psychomécanique formule lors de sa conférence à l'école pratique des hautes études le 23 décembre 1948 permet encore un peu plus de distinguer ce qui relève du domaine de l'institué, celui de la langue, et du domaine du discours, momentané et infini. Le lien entre sujet parlant et signification est ainsi affirmé ainsi que la nature changeante de la signification en fonction des conditions, chaque fois nouvelles, de l'énonciation. Lors de la même conférence, Guillaume explique : «La visée de discours [comporte] partout et toujours le choix d'une partie en convenance particulière avec ce que l'on se propose d'exprimer. ${ }^{11}$ Le choix des mots, en fonction d'un vouloir-dire ou d'un " à-dire, » pour reprendre la terminologie de Lafont en praxématique, est l'opération nécessaire qui suppose l'intervention d'un locuteur réel.

Considérer que Gustave Guillaume conçoit la langue comme le siège de la pensée ou bien que la pensée précède le langage est une simplification. La langue, en tant que domaine puissanciel, n'a pas vocation à exprimer le pensé mais à représenter le pensable. Est pensable ce qui peut être pensé. La langue institue en elle les moyens dont a besoin la pensée pour opérer sa propre saisie, ou, pourrait-on dire, pour prendre une forme

10 La réaction à la linguistique de l'École de Genève dans le contexte du rejet du positivisme est notamment au cœur de la philosophie du langage de l'Ecole de Munich chez Karl Vossler puis chez Volochinov en Russie. À ce sujet, voir la préface de Patrick Sériot à la traduction de Marxisme et philosophie du langage de Volosinov, 2010, p. 74-76

11 «La notion de visée en psychomécanique du langage : essai de définition-2, » site du Fonds Gustave Guillaume, dernière consultation le 30 mai 2020. 
intelligible. Gustave Guillaume dira : «le discours a pour matière d'expression le pensé. La langue a pour matière de représentation le pensable » $(1970,160)$. Si l'on exprime à partir du représenté et que l'on pense à partir du pensable alors la langue est le moyen conditionnant les représentations et l'expression. Cela dit, on sait que les changements en langue sont institués par l'usage, c'est-à-dire par les actualisations en discours. L'une des lois de ce changement est celle de l'économie du langage c'est-à-dire une tendance à adopter dans l'usage les formes les plus commodes à utiliser. On peut se demander alors si l'usage ne conduit pas non seulement à des phénomènes de formules stéréotypées mais aussi si ces formules, une fois instituées, ne conduisent pas à une systématisation de l'expression. Dans ce cas, il est évident que l'acte d'expression, bien que sans cesse renouvelé dans son processus, n'exclut pas certains automatismes hérités des pratiques langagières collectives et répétées. On peut également se demander si le pensable, situé en langue, domaine de l'institué collectivement, ne peut évoluer au gré des formations discursives en circulation. Ou bien, si la parole effective ne saurait modifier le champ du pensable situé dans le système de la langue. On peut alors se demander si les formules discursives stéréotypées relèvent bien d'un processus du pensable au pensé.

\section{LA NOTION DE PAROLE CHEZ ARMAND ROBIN ET JACQUES ELLUL}

\section{PRÉSENTATION D'ARMAND}

ROBIN, LA FAUSSE PAROLE

«Armand Robin a inventé un métier qu'on exerce chez-soi et grâce auquel on peut être transporté dans tous les points du monde où l'on parle. Frais d'installation : un poste de radio. Connaissances exigées : une quinzaine de langues vivantes. ${ }^{12}$

Armand Robin (1912-1961; pseudonyme de Vincent Robin) est tout d'abord un poète et traducteur français. Sa connaissance de la langue russe, dont il déclarera avoir fait sa langue natale au retour d'un voyage en U.R.S.S. en 1933, fut investie dans la traduction des poètes Alexandre Blok (1880-1921), Sergueï Essenine (1895-1925), Vladimir V. Maïakowski (1893-1930), pour ne citer que ceux-là. II traduisit également des textes du poète hongrois Endre Ady de Diósad (1877-1919). C'est à partir de l'année 1941, sous le régime de Vichy, que l'écrivain polyglotte fut engagé par le ministère des affaires étrangères afin d'assurer l'écoute des radios étrangères et fournir un bulletin d'information politique. II occupa ce poste jusqu'en 1943, année de son renvoi après avoir collaboré avec la Résistance. Armand Robin continua cependant son activité d'écoute à titre personnel jusqu'à sa mort en 1961. Il y aurait beaucoup à dire sur le plurilinguisme de Robin, sa relation avec la langue française, langue d'emprunt; sa mise à l'état de vacance pour se fondre dans les paroles de locuteurs éloignés. II s'agira ici de se limiter à la façon dont Armand Robin commente la parole à travers son activité d'écoute. Ces commentaires sont, bien entendu, ceux d'un locuteur dit naiif, ou locuteur profane, c'est-à-dire un locuteur qui n'est pas linguiste de métier. Pour autant, nous voyons là l'occasion de prendre en compte la perception, au sens phénoménologique, de l'essence d'une non-langue, ou plus exactement, d'une non-parole.

L'édition augmentée de 2002 se compose de l'ouvrage initial paru pour la première fois en 1953 aux éditions de Minuit auquel ont été ajoutés trois textes complémentaires dont deux textes écrits après La Fausse parole. L'auteur commence par une remarque. L'ensemble de mots « Le lion mit à sécher son burnous dans la rivière » aussi absurde qu'il paraisse est bien plus sensé que n'importe lequel des discours politiques qui déferlent sur les ondes soviétiques. Aussi l'auteur s'interroge (ibid., 25) : «Pourquoi le poète ou le grammairien sont-ils dans le vrai en proposant des ensembles de mots étranges et pourquoi le politicien, en usant de même avec le langage, se trouve-t-il comme mécaniquement dans l'erreur? Ce mystère vaut d'être éclairci. » La dimension mystérieuse de la fausse parole émaille le texte où il est question de part en part d'emprise psychique, de destruction des consciences, et de mise à mort du Verbe. Pourtant, celui qui écoute ne cherche pas à tomber dans l'irrationnel (ibid., 43) : « Je ne m'exprime pas ainsi par goût pour l'irrationnel ni par tendance à la poétisation. J'essaie de cerner une réalité peu connue, de cerner et de définir un état de fait. » Pour présenter de façon synthétique les caractéristiques de la fausse parole, nous retiendrons deux aspects. En quoi la fausse parole est-elle fausse? La fausse parole est, tout d'abord, une parole sans sujet. Les discours de propagande qui déferlent sur les ondes forment un seul et même texte, une «non-langue de toutes les langues, » qui semble avoir son existence propre (ibid., 57). S'il n'est jamais question d'idéologie dans l'ouvrage c'est que ce sont bien les mots dans leur forme vocalisée qui circulent tout seuls, et non les idées. Les locuteurs sont alors les relais passifs d'une parole anonyme qu'ils répètent de façon automatique. Les propagandistes eux-mêmes n'ont aucune autorité sur leur discours mais sont de simples véhicules d'un flot au mouvement autonome. L'individu n'est plus en possession de son langage et les paroles qu'il prononce sont inhabitées.

Ensuite, la fausse parole est celle qui ne signifie rien. Sans rapport avec le vécu, la fausse parole a pour but de transformer la réalité, de «corriger l'humanité de son indésirable propension à constater que ce qui existe existe. » (p. 72). Robin insiste sur cet aspect de façon

12 Extrait du prière d'insérer de l'édition de La fausse Parole de 1953, cité par Françoise Morvan dans son introduction à l'édition de 1979 et reprise dans la version de 2002, p. 9. F. Morvan a consacré sa thèse de doctorat d'État en littérature française à l'œuvre d'Armand Robin. 
à différencier la fausse parole d'un simple mensonge. La parole mensongère a intérêt à être crédible. Les propos de la propagande que l'auteur entend semblent avoir été « très exactement choisis dans la mesure où l'on sait qu'ils ne seront pas crus. » (ibid., 73, en italique dans le texte original). La fausse parole agit alors comme un puissant facteur d'aliénation mentale et s'inscrit dans une entreprise de « liquidation de l'entendement humain » (ibid., 75). Cependant, Robin observe une forme d'unification des discours au lendemain de la seconde guerre mondiale et remarque (ibid., 91) que la tendance typiquement soviétique s'étend au bloc occidental, et, plus particulièrement, étatsunien :

« Naguère, il n'y avait que la radio soviétique à être aussi muette, aussi inutilement et puissamment robotisée ; aujourd'hui, sur toutes les longueurs d'ondes c'est le même catéchisme mécanisé [...] il s'agit dans les deux cas d'une radio massive, compacte, impénétrable, d'une radio en laquelle aucune authentique parole n'a de chance de pénétrer. ${ }^{13}$

Plus tard, dans un autre texte daté de 1955, l'auteur remarque un net changement de style, tant dans les discours radiophoniques du bloc de l'Est que dans ceux du bloc anglo-saxon. Le ton autoritaire et vindicatif semble avoir disparu depuis la mort de Staline en 1953 et le fanatisme avoir cédé le pas à un ton plus modéré. Si Robin voit dans ce changement un recul stratégique de la terreur face à la menace atomique, il émet également l'hypothèse d'un nouveau modèle politique où la technique et le langage feutré des experts tend à remplacer les modèles hégémoniques surannés. La « détente ${ }^{14}$ qui s'amorce alors traduit le renoncement des deux puissances majeures de l'époque au profit des « Savants absolus. » La mathématique quantitative semble désormais le nouveau paradigme destiné à gouverner les sociétés, y compris les partis et les institutions. Robin note une nette différence entre les deux modèles de potentats (ibid., 101) :

«Les gouvernements [...] connaissaient la valeur des mots sortant du cœur avec amour ou révolte ; ils les écrasaient ou les utilisaient, selon leurs intérêts du moment. Puis eux-mêmes bavardaient sans cesse, tenaient par tout moyen à ce que tous leurs ressortissants bavardent en leur sens. Ceux devant qui les deux plus puissants gouvernements du monde se sont inclinés, ils ne bavardent pas, eux. La parole, vraie ou fausse, ils ne la méprisent même pas : ils ignorent son existence. $»^{15}$
Difficile de ne pas voir dans ces lignes les prémisses d'un nouveau langage aux contours techniques et scientifiques, tel que le décrira Uwe Poerksen à la fin des années 1980 dans le contexte des deux Allemagne. ${ }^{16}$

\section{PRÉSENTATION DE JACQUES ELLUL, LA PAROLE HUMILIÉE (1981)}

L'œuvre de Jacques Ellul (1912-1994) est généralement associée à une certaine critique sociologique et philosophique de la technique dans le monde contemporain. Pourtant, si la réflexion de Jacques Ellul dans ce domaine fut féconde, l'auteur du Système technicien était tout d'abord docteur puis agrégé en Droit. Libertaire, engagé dans la Résistance, Ellul fut parfois qualifié d'anarchiste chrétien tant ses analyses se nourrissaient à la fois de sa foi chrétienne (Ellul était protestant) et d'une réflexion politique sur les conditions de la liberté humaine. De 1954, année de publication de La Technique ou l'enjeu du siècle jusqu'en 1988, année de parution du Bluff technologique, la pensée critique de Jacques Ellul cheminera aux côtés de celle de Bernard Charbonneau. La Parole humiliée, paru en 1981, s'inscrit donc chronologiquement dans la dernière partie de l'œuvre ellulienne. On pourrait voir dans cet essai une réflexion sur la communication - ses nouvelles modalités de mise en œuvre et ses dogmes - réflexion légitime étant donné l'émergence de nouveaux moyens de communication à l'époque et la place croissante du divertissement et de l'information qui avait suscité l'intérêt d'autres auteurs quelques années auparavant. ${ }^{17}$ Toutefois, alors qu'Ellul focalise cette fois sur l'aspect communicationnel de la vie en société, on peut y voir une version synthétique et mise à jour de sa critique du « système technicien » à l'aune d'une réflexion sur le langage. Le ton est donné dès les premières lignes (p. 5) : « Ne cherchons ici ni aucune étude savante sur l'expression iconique, ni la syntagmatique, ni le métalangage. [...] Je suis homme quelconque et je dis ce que vit n'importe qui. Sans chercher de science. » Non seulement l'auteur ne prétend pas se mesurer aux disciplines académiques spécialisées dans le langage mais il affirme la pertinence du sens commun et de l'expérience ordinaire. « Je suis, j'écoute, je regarde, » déclare-t-il, mettant par là en balance l'expérience des sens et la perception de l'homme simple avec la démarche scientifique et ses codes conceptuels. N'eusse été la carrière universitaire d'Ellul, on pourrait voir là une attaque contre une démarche scientifique jugée artificielle. L'auteur cherche cependant davantage à replacer le langage au cœur de l'expérience humaine, et c'est bien en intellectuel, intellectuel engagé certes, qu'il entend aborder ce sujet.

13 A. Robin, La Fausse parole. Mazères, Le temps qu'il fait. 2002 [1953], p. 91.

14 La guerre froide fut ponctuée de plusieurs périodes de relâchement des tensions entre le bloc soviétique et

I’Amérique du Nord. La première couramment identifiée commence en 1953 à la mort de Staline.

15 Robin, 101.

16 U. Poerksen, linguiste allemand, est l'auteur du concept de «mot plastique » (1988) pour désigner les discours politiques en circulation en Allemagne de l'Est et en Allemagne de l'Ouest dans les années 1980.

17 On peut penser notamment à Guy Debord (1967) et Jean Baudrillard (1968). 
Il faut toutefois souligner que la façon dont l'auteur pourfend la linguistique moderne est assez classique. ${ }^{18}$ De plus, la dichotomie modèle/réalité, théorie/expérience, qui remonte à une époque bien antérieure à celle de J. Ellul est emblématique d'un certain scepticisme à l'égard des théories mécanistes des choses humaines et vivantes en général. ${ }^{19}$

L'ouvrage s'articule sur le constat du règne de l'image, d'où s'ensuit le recul de la parole. Recul de l'oralité mais aussi de la parole sous sa forme écrite car ce qui inquiète l'auteur n'est pas tant la forme que le contenu qui change. On retrouve alors des réflexions sur le sens, la subjectivité (bien que non formulée comme telle), la place de l'homme dans son langage.

La parole humiliée correspond tout d'abord à ce qu'Ellul appelle une dévaluation de fait. Le thème du vide, de l'absence est primordial. II s'agit tout d'abord de l'absence de l'être dans sa parole. La déferlante des discours par la voix des médias conduit à une parole anonyme, continuelle, dépersonnalisée et dont les individus forment à la fois le réceptacle et le relai passif. Ellul oppose ainsi la parole incarnée par un sujet et la nouvelle parole, plurielle car collective et cependant unifiée dans un ensemble conceptuel homogène. Ellul examine également le langage conçu comme instrument en corrélation avec le modèle de l'ordinateur : un langage sans ambigüité ni complexité. Enfin, l'auteur identifie deux pratiques différentes d'où résulte «l'éclatement du langage : » celle du langage technicien dépourvu des ambivalences et des incertitudes du langage ordinaire. On reconnaît là une critique de la vision utilitariste du langage inspiré du modèle de la communication et ses notions d'émetteur, récepteur et de message. La deuxième pratique est celle des intellectuels et des artistes déconstructivistes et surréalistes qui, à force de se jouer du sens, ont font une bagatelle et traitent l'expression langagière comme une pure forme valant pour elle-même.

\section{SYNTHĖSE}

La Fausse parole et la Parole humiliée furent écrits à des époques différentes éloignées de plus de trente ans. Les deux ouvrages diffèrent également par le genre littéraire dans lequel ils s'inscrivent: journal dans le cas de la Fausse parole et essai dans le cas de La Parole humiliée. Si le premier peut être considéré comme un document historique, le second est à vocation critique et polémique. Néanmoins, les deux ouvrages ont cela de commun qu'ils ont pour auteurs des écrivains, c'est-à-dire des disciples du langage pour qui celui-ci est à la fois une praxis et une condition de l'existence de l'être. Les contextes dans lequel ils prennent naissance, éloignés sur le plan chronologique, sont assez différents. Celui de la Parole humiliée est le règne soviétique, situé à l'Est, alors que celui d'Ellul est celui du règne occidental, situé en Europe et aux États-Unis en particulier. Néanmoins, les deux ouvrages ont en commun d'appartenir à deux époques de transition sociologique. Armand Robin écrit à l'époque d'un monde encore marqué par la bipolarité Est-Ouest glissant vers la dynamique multipolaire amorcée au sortir de la seconde guerre mondiale. La mutation sociale réside aussi dans le vécu du régime totalitaire, tant au niveau de la vie en société et de la vie intérieure qu'au niveau de la menace d'une destruction totale par la technologie nucléaire. Les réflexions de Jacques Ellul interviennent à un moment d'accélération de la technique et du modèle consumériste, nouveaux avatars d'une volonté de puissance et d'une vision englobante de l'humanité civilisée. Ce qu'Armand Robin tente d'appréhender dans la plus grande partie de l'ouvrage, c'est le langage d'une époque marquée par les régimes autoritaires en URSS et en Occident alors que l'ouvrage d'Ellul parait à une époque débarrassée de ces modèles autoritaires et coercitifs.

L'intérêt commun pour la parole de la part de deux écrivains à deux époques différentes de mutation sociale montre au moins deux choses : d'une part, l'expansion d'un modèle social émanant d'un système politico-économique a des répercussions sur la vie du langage. Les écrivains semblent tout particulièrement dotés pour décrire et penser ces mutations. ${ }^{20}$ Cependant, la façon dont ces auteurs abordent la question du langage reste en dehors du cadre scientifique linguistique. La parole dont parlent Robin et Ellul n'est pas la parole au sens structuraliste. Elle ne se situe pas dans une relation paradigmatique d'opposition à la langue mais plutôt dans une relation d'opposition au discours. Nous retiendrons quelques traits distinctifs de cette opposition.

La parole est indissociable du sujet individuel. Le discours peut être collectif et anonyme alors que la parole est un acte nécessairement individuel et auctorial. Par exemple, on peut comprendre une expression telle que « le discours du pouvoir » pour faire référence à la manifestation verbale et homogène d'une vision politique portée par un gouvernement mais on ne saurait voir dans l'expression « la parole du pouvoir » un sens similaire. De plus, le discours est reproductible alors que la parole est chaque fois réitérée. II est possible de s'approprier un discours et le répéter mais il est difficile de parler de parole dans ce cas, à moins de décliner ce mot au pluriel. On dira alors répéter le discours de quelqu'un ou bien les

18 On retrouvera notamment ce thème dans l'essai de Jaime Semprun, Défense et illustration de la novlangue française, Éditions de l'encyclopédie des nuisances, 2005.

19 K. Vossler (1872-1949) écrivait déjà, en 1904 : La parole vivante était disloquée en phrases, membres de proposition, mots, syllabes et sons » (voir K. Vossler, 1904, p. 8 cité par P. Sériot, 2010, p.75).

20 On pense à Aleksander Wat (1900-1967) et Czesław Miłosz (1911-2004) mais aussi à Jaime Semprun et à de nombreux journalistes tels Karl Kraus (1874-1936), Dolf Stenberger (1907-1989), Eric A. Blair alias George Orwell (1903-1950). 
paroles de quelqu'un mais pas «la parole de quelqu'un » car la parole s'inscrit dans un moment singulier d'expression. Ainsi, le mot parole revêt des sens différents selon qu'il est employé au singulier, forme qui nous intéresse ici, ou au pluriel, où son sens est plus proche de celui de discours comme dans les tours suivants:

«Ses paroles n'ont aucun sens. »

«Sa parole n'a aucun sens. »

Si langue et parole sont indissociables, parole et discours peuvent être distingués et ne cohabitent pas forcément. Le discours sans parole est le discours où toute forme de dialogisme s'efface. C'est un discours sans sujet, ou bien, dans le cas d'un discours dont l'identité de l'auteur est définie, un discours que la pensée du sujet a désinvesti. La parole est un acte de langage alors que le discours est un acte de communication où les mots servent de matière verbale. On peut se dissocier d'un discours, pas de sa parole. La parole est indissociable de l'être. En somme, la parole suppose l'intériorité alors que le discours suppose l'extériorité.

Comparons les exemples suivants:

(i) Son discours est ambigu.

(ii) Sa parole est ambiguë.

(iii) C'est mon discours.

(iv) C'est ma parole.

Dans les exemples (i) et (ii), si l'ambiguïté peut être attribuée à un discours, il est difficile de l'attribuer à la parole, lieu de la subjectivité où locuteur et dire sont indissociables.

Dans l'exemple (iii), on conçoit une possible distanciation entre l'énonciateur et son discours et on pourrait gloser par «c'est le discours que je tiens » sans que l'énonciateur adhère nécessairement à ce qu'il dit, ni qu'il en soit l'instigateur.

Dans l'exemple (iv), au contraire, on voit nettement le caractère performatif d'un tel énoncé où « ma parole » ne saurait être celle de quelqu'un d'autre que l'on s'approprie pour la répéter. Le locuteur et la parole sont ici indissociables.

Quand Ellul et Robin parlent de parole ils ne font pas la critique d'un discours, ni une critique de la langue mais bien de la parole en tant que lieu d'expression verbale de l'individu. La parole, dans ce cas, n'est pas seulement un acte de communication comme le serait un discours ni un acte phonatoire au sens saussurien mais un acte où se conjuguent l'être et le langage, soit en figure :

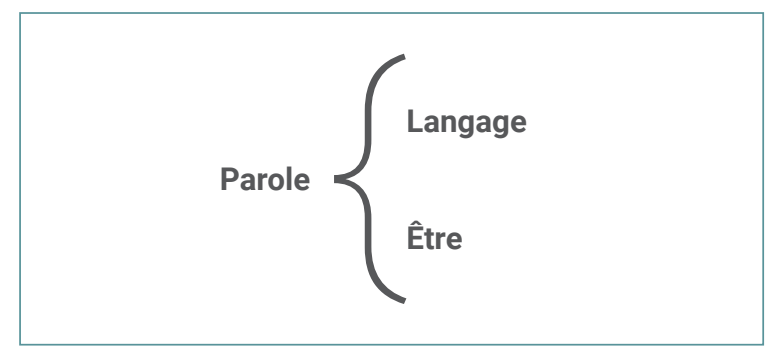

Figure : relation entre être, langage, et parole
Une telle relation entre être, langage, et parole suppose la singularité de l'expression ainsi que la dimension dialectique entre sujet et réalité. La singularité de l'expression tient au fait que chaque locuteur étant unique auteur de sa parole, celle-ci ne saurait être interprétée de la même manière que celle de quelqu'un d'autre. La dimension dialectique entre sujet et réalité suppose le nécessaire dialogue entre l'être parlant et le monde dont il fait l'expérience, dialogue où siège l'interprétation et la signification. C'est pourquoi la parole ne saurait se résumer au discours collectif, ni à la production orale d'énoncés.

Pour terminer, et puisque la parole, dans le sens que nous avons expliqué, ne s'oppose pas à la langue mais davantage au discours, on peut envisager sous un jour nouveau l'expression langue de bois employée pour parler de ce qu'il conviendrait d'appeler un discours. II manque probablement une vraie parole à la langue de bois. La parole étant liée à l'intériorité, tout comme la langue, dont elle dépend, il se peut que le terme langue dans langue de bois soit entendu au sens de parole. Aussi fausse parole et langue de bois ne sont qu'un seul phénomène vu sous des angles différents. La parole étant nécessairement singulière, le terme langue aura naturellement mieux trouvé sa place pour désigner la manifestation d'une pensée collective. L'homogénéité des concepts, la répétition des tropes et la présence d'une idéologie, c'est-à-dire d'une pensée, fût-elle unique et anonyme, a pu donner l'impression d'un système préalable au discours en tant que tel. Lorsque la parole est absente du discours, il semble ne rester que la langue, c'est-à-dire le système lexico-syntaxique propre à former des propositions. La langue de bois serait alors un discours sans parole.

La notion de parole, chez Ellul et Robin, nous entraîne en dehors du champ de la linguistique en tant que science modélisatrice. Chaque sujet parlant étant unique, la parole, lieu de l'expressivité et lieu momentané, ne saurait faire l'objet d'un modèle général. Dans sa version silencieuse, la parole intérieure ne saurait être abordée que par l'introspection et est encore plus éloignée du champ de la description linguistique située en discours. La notion de parole est en revanche utile à déterminer ce qu'est une non-parole, par-delà l'apparence des discours formulés.

\section{CONCLUSION}

La notion de parole nous emmène loin des conceptions de la langue comme instrument et fait le pont entre le système institué de la langue, condition du pensable, et la signification, domaine de l'actualisé. La psychosystématique du langage de Gustave Guillaume, tout d'abord, permet de dépasser la dichotomie langue-parole et de prendre en compte l'activité du locuteur de la pensée jusqu'au discours. Avec Armand Robin et Jacques Ellul, nous avons voulu montrer deux réflexions sur la parole à deux époques et dans deux contextes différents. Qu'il s'agisse d'une activité d'écoute radiophonique à l'époque de la Guerre Froide ou d'une approche philosophique en lien avec la croissance technologique, ces deux lectures 
montrent des constantes dans l'analyse du langage ambiant à l'ère postindustrielle. Le mot parole vient alors remplacer celui de langue pour faire référence à ce qui est traditionnellement qualifié de langue de bois dans son sens fort. Nous avons dit que la parole était nécessairement intérieure. En employant le mot parole au lieu de langue ou discours, l'accent est mis sur le monde intérieur du sujet-parlant plutôt que sur la notion de système ou d'expression.

Parole humiliée, fausse parole, sont des façons de nommer la langue de bois dans son sens fort qui dépassent, avec les théories linguistiques classiques, la dichotomie langue-discours dans l'analyse de la langue de bois. La langue de bois serait alors non pas la manifestation d'un non-dit, au sens où l'expression sincère ne peut avoir lieu, mais d'un non-dire, au sens où ni le sujet, ni la réalité ne permettent d'inscrire la langue de bois dans l'univers de l'expérience des faits.

\section{RÉFÉRENCES BIBLIOGRAPHIQUES}

Bally, C., 1965 [1913]. Le langage et la vie. Librairie Droz. Genève.

Bally, C., 1922. La Pensée et la langue. Bulletin de la société linguistique de Paris, vol. 3, p. 117.

Baudrillard, J., 1968. Le Système des objets : la consommation des signes. Paris, Gallimard.

Benveniste, E., 1966. Problèmes de linguistique générale, vol. 1. Paris, Gallimard.

Boone A. et Joly A., 2002. Dictionnaire terminologique de la systématique du langage. Paris, L'Harmattan.

Calvet, L.-J., 2017. La Sociolinguistique. Paris, PUF.

Charaudeau, P., 2015. De la linguistique de la langue à la linguistique du discours, et retour. Engwall, Gunnel \& Fant, Lars (eds.) Festival Romanistica. Contribuciones lingüísticas - Contributions linguistiques - Contributi linguistici - Contribuições linguísticas. Stockholm Studies in Romance Languages, Stockholm: Stockholm University Press, p. 3-12.

Culioli A., 1991. Pour une linguistique de l'énonciation. Paris, Ophrys.

Debord G., 1967. La Société du spectacle. Paris, Buchet-Chastel.
Ducrot, O., 1984. Le Dire et le dit. Paris, Les Éditions de Minuit.

Ellul, J., 1981. La Parole humiliée. Paris, Seuil.

Guillaume, G., 1971. Leçons de linguistique, vol. 8. Québec, Presses de l'Université Laval.

Guillaume, G., 1970. Principes de linguistique théorique. Québec, Presses universitaires de Laval; Paris, Klincksieck.

Guillaume, G., 1965 [1943]. Temps et verbe suivi de Architectonique du temps dans les langues classiques. Paris, H. Champion.

Joly, A., 1987. Systématique énonciative. Lille, Presses Universitaires de Lille.

Lafont, R., 1983. L'à-dire et le temps du silence: pour une linguistique de la parole productrice. Cahiers de praxématique, 1, p. 10-44.

Monneret, P., 2003. Notions de neurolinguistique théorique. Dijon, Presses universitaires de Dijon.

Morvan, F., 1989. Armand Robin : bilans d'une recherche. PhD thesis, Université Rennes 2.

Poerksen, U., 1995. Plastic words. The Tyranny of a Modular Language. Pennsylvannia, Pennsylvannia University Press.

Poerksen, U., 1988. Plastikwörter : die Sprache einer internationalen Diktatur. Stuttgart, Klett-Cotta.

Robin, A., 2002 [1953]. La Fausse parole. Mazères, Le temps qu'il fait.

Saussure, L.-F., 1971 [1916]. Cours de linguistique générale. Paris, Payot.

Semprun, J., 2005. Défense et illustration de la novlangue française. Paris, Éditions de l'encyclopédie des nuisances.

Sériot, P., 2010. Préface in Volochinov V. N.. Marxisme et philosophie du langage. Limoges, Lambert-Lucas.

Tollis, F., 2008. La linguistique de Gustave Guillaume : de l'opérativité à la socio-opérativité ?, Cahiers de praxématique, 51, p. 131-154.

Valette, M., 2003. Énonciation et cognition : deux termes in absentia pour des notions omniprésentes dans l'œuvre de Guillaume. Le Français moderne, 1, p. 6-25. 